\title{
Perfil Psicomotor en Niños Escolares: Diferencias de Género
}

\section{Psychomotor Profile in School Children: Gender Differences}

Recibido 1 Jun. 2013/Enviado para Modificación 30 Sep. 2013/Aceptado 18 Oct. 2013

\author{
Luz Mery Noguera Machacón ${ }^{1}$ \\ Universidad Simón Bolívar
}

\author{
Floralinda García Puello
}

Universidad Simón Bolívar

\section{RESUMEN}

Introducción: El período de vida comprendido entre los 4 y los 8 años de edad resulta de gran importancia para el desarrollo de las condiciones y habilidades motrices de los niños, que son necesarias para la realización efectiva de actividades en el resto de la vida adulta. Objetivo: Describir el estado del perfil psicomotor según el género en la población escolar comprendida entre los 4 y 8 años de edad. Materiales y Método: Estudio de tipo descriptivo transversal en 389 niños y niñas, que se desempeñan como estudiantes en 8 instituciones educativas públicas de la ciudad de Barranquilla y del municipio de Puerto Colombia. Para determinar el perfil psicomotor de los niños, se utilizó la batería de Vítor Da Fonseca, y luego se realizó un análisis bivariado entre las medias del perfil motriz y su comportamiento según el género. Resultados: En cada una de las unidades motrices valoradas, se encontraron diferencias significativas entre niños y niñas. El equilibrio $(44,9 \%)$ y la praxia fina $(44,9 \%)$ obtuvieron mejores puntajes en las niñas; pero, en cuanto a la estructuración espacio-temporal, las niñas fueron categorizadas en su mayoría como deficitarias y dispráxicas. Conclusión: En cada una de las unidades motrices valoradas, se encontraron diferencias significativas entre niños y niñas; por lo tanto, los programas de intervención para la estimulación motriz deben ser individualizados de acuerdo con las necesidades particulares de la población.

Palabras Clave: Desempeño Psicomotor, Salud Escolar, Habilidades Motoras, Género. (Fuente: DeCS).

\begin{abstract}
Introduction: The period children's lives from ages 4 to 8 years old is very important for their motor conditions and skills, which are necessary for physical performance during the rest of their adult lives. Objective: To describe the psycho-motor profile according to the gender of the school population of children ages 4 to 8 years old. Materials and Methods: A transversal and descriptive study using a sample of 389 children, who are students from different public schools from Barranquilla and Puerto Colombian town. The Vitor Da Fonseca Psycho motor battery was used to determine the psycho-motor profile in children and later a bivariated analysis between children's psycho-motor profile and their behavior by gender. Results: In each evaluated unit, significant differences between boys and girls were found. The balance $(44,9 \%)$ and the fine $(44,9 \%)$ practice showed better results in girls. Based on the temporal-spatial structure, the girls were classified mostly as loss/making and showed dyspraxia. Conclusion: In each of the psychomotor units assessed, significant differences were found between boys and girls, so the intervention programs for psycho-motor stimulation must be individualized according to the specific needs of the population.
\end{abstract}

Keywords: Psychomotor Performance, School Health, Motor Skills, Gender (Source: MeSH, NLM).

1 Fisioterapeuta. Magister en Educación. Universidad Simón Bolívar, Barranquilla. Correo electrónico: Inoguera1@ unisimonbolivar.edu.

2 Fisioterapeuta. Estudios en medicina tradicional china. Especialista en fisioterapia en ortopedia. Maestrante en Salud Pública. Correo electrónico: fgarcia@unisimonbolivar.edu.co 


\section{Introducción}

El desarrollo psicomotor es un proceso evolutivo, multidimensional e integral, gracias al cual, el individuo logra dominar progresivamente habilidades y respuestas cada vez más complejas, cuyo objetivo final es la adquisición de independencia, fortalecimiento de la personalidad y la capacidad de interactuar con el mundo y transformarlo. Este proceso comienza desde la vida intrauterina y se fortalece en la etapa de la edad escolar, cuando se forjan de igual forma el carácter y la conducta del niño (1).

En esta etapa, el juego constituye un recurso fundamental para estimular el desarrollo de la motricidad y, por ende, el fortalecimiento de las capacidades cognitivas, sociales y lingüísticas de los niños. Para algunos autores, la adquisición de nuevas habilidades motrices en la edad escolar constituye la "Edad de oro del Aprendizaje Motor", debido a la facilidad que tienen los niños durante esta etapa para el aprendizaje de diferentes habilidades motrices (2).

El desarrollo motor humano atraviesa por fases en las que la característica principal es el tipo de movimiento coordinado manifestado a través de movimientos reflejos, rudimentarios, fundamentales, específicos y especializados. La primera fase es de carácter preferentemente cualitativo y comprende la organización psicomotora y la estructuración de la imagen corporal. Está compuesta por una serie de estadios en los que el cuerpo atraviesa sucesivas transformaciones (cuerpo impulsivo, vivido, percibido y representado). La segunda fase se corresponde con las transformaciones musculares y las mejoras en el rendimiento motor, que se traduce en los denominados factores de ejecución (3).

Son múltiples las razones que motivan a estudiar el desarrollo motor y sus fases. De un lado, existe la inquietud por determinar la correspondencia entre la edad cronológica de los niños y las competencias motrices que poseen; además de las diferentes características que influyen en la eficacia o no del conjunto de movimientos; $y$, por otro, como en nuestro caso en particular, aparece la necesidad de identificar las diferencias motrices en los niños según el género. Esto último amplía el abanico de posibilidades de intervención en la educación y en la estimulación del desarrollo psicomotor de los niños con base en sus reales necesidades e intereses. De igual forma, un adecuado estímulo de la psicomotricidad permitirá el desarrollo apropiado de las posibilidades intelectivas del niño que requieren la intervención del movimiento (4).

Para todo esto, resulta fundamental aprovechar la gran riqueza de conductas motrices que poseen los niños en la edad escolar, etapa en que, a través del juego, ponen en marcha toda su capacidad motriz para afrontar sus propios problemas y, al mismo tiempo, van conformando su personalidad y modos de conducta (5).

La batería de Vítor Da Fonseca constituye un referente apropiado para el tema de la presente investigación, ya que permite establecer de forma cuantitativa las conductas atípicas presentes en el desarrollo motriz de la población infantil y, de este modo, ayuda a establecer las diferentes problemáticas en el movimiento y el aprendizaje. Los resultados de su aplicación evidencian, en efecto, las deficiencias puntuales en la adquisición de habilidades motrices tanto de los niños como de las niñas.

\section{Materiales y Métodos}

Se realizó un estudio de tipo descriptivo transversal en 389 niños y niñas de 4 a 8 años de edad, que cursaban estudios en siete instituciones educativas públicas de la ciudad de Barranquilla y en una del municipio de Puerto Colombia, Atlántico. La media de edad fue de 5.5 años. Además, el $60.2 \%$ de la población estuvo constituido por niñas, en tanto que el $32.9 \%$ cursaba el grado de Transición.

Como se ha reiterado, en esta investigación se utilizó la Batería Psicomotora (BPM) de Vítor Da Fonseca (6). Esta se ha utilizado ampliamente como un sistema de observación de los diversos componentes del sistema motor; los datos obtenidos a través de ella reflejan el grado de organización neurológica del niño, lo que posibilita identificar alteraciones de forma eficaz. En concreto, un estudio realizado en la ciudad de Manizales, Colombia, donde se evaluaron 846 niños, da cuenta de la fiabilidad de la aplicación de esta batería para identificar trastornos en la motricidad de niños en edad escolar (7). Asimismo, cabe resaltar que al realizar el análisis de consistencia interna de la batería, el Alfa de Cronbach arrojó un resultado de 0,51 ; que es un valor aceptable de confiablidad estadística.

Cienc. innov. salud. Diciembre 2013; 1 (2):108 - 113. Universidad Simón Bolívar (Col). ISSN: 2344-8636 http://portal.unisimonbolivar.edu.co:82/rdigital/innovacionsalud 
Este instrumento consiste en una serie de pruebas que evalúan 7 áreas o subfactores de la psicomotricidad y permiten describir el perfil psicomotor del niño. Cada subfactor asigna una puntuación de 1 a 4 puntos, donde 1 punto, apraxia, corresponde a la ausencia de respuesta, o a una realización imperfecta, incompleta, inadecuada $\mathrm{y}$ descoordinada; 2 puntos, dispraxia, sugiere una realización débil con dificultades de control y señales desviadas; 3 puntos, eupraxia, indican que la realización de la actividad es completa, adecuada y controlada; y 4, hiperpraxia, que la realización es perfecta, precisa, económica y con facilidad de control. El perfil psicomotor se obtiene mediante la sumatoria de los promedios de los siete factores $\mathrm{o}$ capacidades motrices.

Con la aplicación de este instrumento se caracterizan así las potencialidades y dificultades de los niños, y se pueden identificar e intervenir problemas de aprendizaje psicomotor.

Por otra parte, el período de evaluación fue de 30-40 minutos (evaluador capacitado) y los materiales utilizados fueron de bajo costo y sin ningún tipo de sofisticación. Se utilizó el programa estadístico SPSS versión 18, con el cual se establecieron los análisis univariados y bivariados estimados entre las medias del perfil motriz, así como su comportamiento según el género de los participantes.

\section{Resultados}

La población de estudio estuvo compuesta por 389 niños y niñas de 4 a 8 años de edad, de los cuales el $60,2 \%$ era de sexo femenino, y el $39,8 \%$ de sexo masculino. La edad promedio fue de 5,5 $\pm 1,2$ años. El $32,9 \%$ cursaba el grado de Transición, un 23,9\%, el de Jardín, y otro $23,9 \%$. El resto de la población se distribuyó entre los grados segundo y tercero (ver Tabla 1).

Tabla 1. Características sociodemográficas de la población

\begin{tabular}{lll}
\hline & VARIABLES & \multicolumn{1}{c}{ FRECUENCIA (\%) } \\
\hline \multirow{2}{*}{ SEXO } & Femenino & $234(60,2)$ \\
\hdashline & Masculino & $155(39,8)$ \\
\hline \multirow{4}{*}{ CURSO } & Jardín & $93(23,9)$ \\
& Primero & $93(23,9)$ \\
& Segundo & $63(16,2)$ \\
& Tercero & $12(3,1)$ \\
& Transición & $128(32,9)$ \\
\hline-
\end{tabular}

\begin{tabular}{|c|c|c|c|}
\hline \multicolumn{2}{|c|}{ VARIABLES } & \multicolumn{2}{|c|}{ FRECUENCIA (\%) } \\
\hline \multicolumn{2}{|c|}{ MEDIA DE EDAD (DE) } & \multicolumn{2}{|c|}{$5,5(1,2)$} \\
\hline \multicolumn{2}{|l|}{ RANGO DE EDAD } & \multicolumn{2}{|l|}{ 4-8 años } \\
\hline \multicolumn{4}{|c|}{$\begin{array}{l}\text { En relación con el análisis de los factores } \\
\text { psicomotrices según el género, se observaron } \\
\text { diferencias significativas. Un aspecto relevante fue el } \\
\text { hecho de que ninguna niña fue categorizada como } \\
\text { deficitaria o dispráxica (ver Tabla 2). }\end{array}$} \\
\hline \multicolumn{4}{|c|}{ Tabla 2. Factores psicomotrices según género } \\
\hline VARIABLES & & $\begin{array}{l}\text { GENERO } \\
\text { MASCULINO } \\
\text { n (\%) }\end{array}$ & $\begin{array}{l}\text { GENERO } \\
\text { FEMENINO } \\
\mathrm{n}(\%\end{array}$ \\
\hline EQUILIBRIO & $\begin{array}{l}\text { Apraxia } \\
\text { Dispraxia } \\
\text { Eupraxia } \\
\text { Hiperpraxia } \\
\end{array}$ & $\begin{array}{l}10(6,5 \%) \\
63(40,6 \%) \\
62(40,0 \%) \\
20(12,9 \%)\end{array}$ & $\begin{array}{l}5(2,1 \%) \\
75(32,1 \%) \\
105(44,9 \%) \\
49(20,9 \%)\end{array}$ \\
\hline $\begin{array}{l}\text { ESTRUCTURACIÓN } \\
\text { ESPACIO- } \\
\text { TEMPORAL }\end{array}$ & $\begin{array}{l}\text { Apraxia } \\
\text { Dispraxia } \\
\text { Eupraxia } \\
\text { Hiperpraxia }\end{array}$ & $\begin{array}{l}8(5,2 \%) \\
53(34,2 \%) \\
87(56,1 \%) \\
7(4,5 \%)\end{array}$ & $\begin{array}{l}16(6,8 \%) \\
77(32,9 \%) \\
111(47,4 \%) \\
30(12,8 \%)\end{array}$ \\
\hline LATERALIDAD & $\begin{array}{l}\text { Apraxia } \\
\text { Dispraxia } \\
\text { Eupraxia } \\
\text { Hiperpraxia } \\
\end{array}$ & $\begin{array}{l}0 \\
11(7,1 \%) \\
71(45,8 \%) \\
73(47,1 \%)\end{array}$ & $\begin{array}{l}24(10,3 \%) \\
109(46,6 \%) \\
101(43,2 \%)\end{array}$ \\
\hline $\begin{array}{l}\text { NOCIÓN DE } \\
\text { CUERPO }\end{array}$ & $\begin{array}{l}\text { Apraxia } \\
\text { Dispraxia } \\
\text { Eupraxia } \\
\text { Hiperpraxia } \\
\end{array}$ & $\begin{array}{l}3(1,9 \%) \\
45(29,0 \%) \\
91(58,7 \%) \\
16(10,3 \%)\end{array}$ & $\begin{array}{l}1(0,4 \%) \\
34(14,5 \%) \\
152(65,0 \%) \\
47(20,1 \%)\end{array}$ \\
\hline PRAXIA FINA & $\begin{array}{l}\text { Apraxia } \\
\text { Dispraxia } \\
\text { Eupraxia } \\
\text { Hiperpraxia }\end{array}$ & $\begin{array}{l}20(12,9 \%) \\
73(47,1 \%) \\
52(33,5 \%) \\
10(6,5 \%)\end{array}$ & $\begin{array}{l}15(6,4 \%) \\
99(42,3 \%) \\
105(44,9 \%) \\
15(6,4 \%)\end{array}$ \\
\hline PRAXIA GLOBAL & $\begin{array}{l}\text { Apraxia } \\
\text { Dispraxia } \\
\text { Eupraxia } \\
\text { Hiperpraxia }\end{array}$ & $\begin{array}{l}1(0,6 \%) \\
64(41,3 \%) \\
85(54,8 \%) \\
5(3,2 \%)\end{array}$ & $\begin{array}{l}5(2,1 \%) \\
111(47,4 \%) \\
108(46,2 \%) \\
10(4,3 \%)\end{array}$ \\
\hline TONICIDAD & $\begin{array}{l}\text { Apraxia } \\
\text { Dispraxia } \\
\text { Eupraxia } \\
\text { Hiperpraxia }\end{array}$ & $\begin{array}{l}2(1,3 \%) \\
37(23,9 \%) \\
116(74,8 \%) \\
0\end{array}$ & $\begin{array}{l}0 \\
26(11,1 \%) \\
188(80,3 \%) \\
20(8,5 \%)\end{array}$ \\
\hline TOTAL & & $155(100,0 \%)$ & $\begin{array}{l}234 \\
(100,0 \%)\end{array}$ \\
\hline
\end{tabular}

En general, los resultados arrojados por nuestro estudio evidencian que en cada una de las unidades motrices valoradas existen diferencias significativas entre niños y niñas. En relación con los niños, se observa que en el subfactor equilibrio, el mayor porcentaje de estos $(40,6 \%)$ obtuvo una calificación de dispraxia, es decir, que en su caso la realización de la prueba fue débil, presentando dificultades de control y señales desviadas. Con respecto a la estructuración espacio-temporal, el 56,1\% obtuvo una calificación de 3 , es decir, alcanzando el nivel de la eupraxia, lo cual sugiere que la realización de la actividad fue completa, adecuada y controlada. De modo similar, los subfacores de noción del cuerpo, praxia global y

Cienc. innov. salud. Diciembre 2013; 1 (2):108-113. Universidad Simón Bolívar (Col). ISSN: 2344-8636 
tonicidad presentaron una calificación de 3 en la mayoría de los casos. En cuanto a la lateralidad, el mayor porcentaje de los niños $(47,1 \%)$ obtuvo una calificación de 4, es decir, de hiperpraxia. Ello indica que, en este aspecto, la realización de la prueba por parte del niño fue perfecta, precisa, económica y con facilidad de control. Por último, se observa que la mayor parte de los niños $(47,1 \%)$ obtuvo una calificación de 2 en el subfactor praxia fina, lo que se traduce en dispraxia, es decir, en una realización de la actividad débil, con dificultades de control y señales desviadas.

En cuanto a los resultados de las niñas, se observa que en los subfactores de equilibrio, estructuración espacio-temporal, lateralidad, noción del cuerpo, praxia fina y tonicidad, la calificación de la prueba arrojó una nota de 3, alcanzando el nivel de eupraxia, lo cual significa que la realización de la actividad fue completa, adecuada y controlada. En el caso del subfactor de praxia global, la calificación obtenida por la mayoría de las niñas $(47,4 \%)$ fue de 2 . Esto sugiere la presencia de dispraxia, de modo que la realización de la actividad se observó débil, con dificultades de control y señales desviadas.

En síntesis, en los resultados arrojados por la prueba, se observó que el equilibrio y la praxia fina obtuvieron mejores puntajes en las niñas; y en la estructuración espacio-temporal, las niñas fueron categorizadas, según el puntaje, como apráxicas en mayor porcentaje que los niños. Lo contrario ocurrió en los resultados de los niños, en cuanto a los factores de tonicidad y noción del cuerpo, donde un mayor número de estos obtuvo calificaciones de apraxia.

Ahora bien, en praxia global, una mayor cantidad de niñas obtuvo un puntaje apráxico y dispráxico; y en lateralidad, niños y niñas obtuvieron en su mayoría notas normales y muy buenas. Ninguno de los géneros presentó notas apráxicas; sin embargo, en la categoría de deficitario o dispráxico, se evidenció un mayor porcentaje en las niñas.

\section{Discusión}

Un estudio realizado en España coincide con los resultados arrojados en esta investigación con respecto al subfactor equilibrio. Aunque en tal caso, se utilizó un instrumento de valoración diferente (Movement $\mathrm{ABC})$, se hallaron diferencias significativas en el equilibrio dinámico, que mostraban mejores calificaciones en las niñas que en los niños (8).

Otro subfactor en el que las niñas superaron a los niños de manera general fue la praxia fina. Esta cualidad biomotriz implica la capacidad del niño para agarrar y manipular objetos con cierta precisión (7). Una de las razones que explicarían un mejor rendimiento en este aspecto en las niñas, es el tipo de juegos que se escogen y se les asignan. En general, los niños son más activos y les gusta explorar y manipular objetos más que a las niñas; pero las niñas son más sensibles a las condiciones del medio ambiente, de tal forma que realizan juegos menos activos y dedican menor tiempo a estas prácticas en comparación con los niños. De igual forma, los padres son más permisivos con los hijos varones, lo que se traduce en brindarles mayores oportunidades de momentos activos en su quehacer diario (9).

Desde la edad preescolar, los niños y niñas tienden a realizar diversos tipos de actividades lúdicas y a utilizar variados tipos de juguetes, mostrando diferentes intereses. Los niños se interesan más por juguetes de construcción, de transporte, objetos que pueden manipularse y, en general, de juegos al aire libre, utilizando más espacio físico y practicando juegos más bruscos y de mayor agresividad física que las niñas. Por el contrario, las niñas prefieren jugar a las muñecas, a los disfraces, a representar y simular los trabajos domésticos, jugar a las casitas, leer o dibujar; actividades que potencian la motricidad fina (10).

No obstante lo anterior, los resultados varían en diversas investigaciones. Un estudio realizado en Brasil evidenció diferencias estadísticamente significativas para la motricidad fina y gruesa entre niños y niñas, mostrando mejores calificaciones en los niños para ambas cualidades (11). Por el contrario, un estudio realizado en Barranquilla, evidenció que los niños muestran un menor desempeño en comparación con las niñas para la realización de actividades de motricidad fina y menor capacidad para integrar la coordinación muscular y las habilidades perceptivas en actividades concretas (12), .

Por otro lado, los resultados de esta investigación muestran que los niños tienen mayor control en la estructuración espacio-temporal que las niñas. En este sentido, se pone de manifiesto que ciertas habilidades motrices se desarrollan de forma variable según el género, no porque existan diferencias en cuanto a 
posibilidades motrices, pues los aspectos locomotivos y manipulativos son los mismos, sino porque las condiciones medioambientales y culturales pueden restringir la maduración adecuada de ciertas cualidades psicomotrices. Por esto, las niñas adquieren menor capacidad espacial y más habilidades artísticas; mientras que los niños se desarrollan más en el deporte, dominan el espacio y desarrollan la fuerza.

Estudios científicos confirman que el género condiciona la movilidad espacial en el desarrollo psicocomotríz de los niños. Los niños, en efecto, utilizan el doble del espacio disponible en un determinado contexto que las niñas. También debe tenerse en cuenta que el espacio es un elemento de socialización, y que los estereotipos sexuales de las diferentes culturas limitan y condicionan considerablemente la movilidad espacial de las niñas (13). Asimismo, algunos autores enfatizan en la "significación diferencial en los estereotipos de conducta por género", lo cual alude a las diferencias evidentes que existen entre los comportamientos y actitudes de niños y niñas, que se expresan inevitablemente a través de determinados patrones de movimiento.

Tomando como referencia la estructuración espaciotemporal, la coordinación visomotora y el esquema corporal, otros estudios plantean que las diferencias motrices entre niños y niñas pueden ser verdaderamente significativas si se analizan en función de la inteligencia o desarrollo de la capacidad cognitiva (14).

Los resultados en el subfactor de tonicidad, entendido como el grado de tensión necesario para realizar cualquier movimiento según nuevas situaciones, y desarrollar un equilibrio tónico necesario para experimentar sensaciones en diferentes posiciones y actitudes tanto estáticas como dinámicas (5), muestran menores puntajes en los niños que en las niñas. Estos resultados, así como los encontrados en otros estudios, evidencian la necesidad de ampliar el enfoque de investigación en el campo de la motricidad infantil; sobre todo, para avanzar en la identificación de los factores que influyen en la estimulación o inhibición de la misma, donde el ambiente desempeña un papel importante. Entre estos factores, el sistema educacional puede ser considerado como el medio más apropiado para desarrollar la capacidad creativa y, por consiguiente, la motricidad del niño, dada la gran cobertura que posee y la cantidad de tiempo que los niños le dedican (15).

De acuerdo con los resultados de esta investigación, queda clara la importancia que tiene la estimulación de todas las capacidades físicas, afectivas, intelectuales y sociales del niño; de tal forma que los programas de intervención educativos con base en actividades que desarrollen la motricidad contribuirán ampliamente a ello (16). En este mismo sentido, se puede afirmar que la adquisición de las cualidades motrices en los niños está íntimamente relacionada con el pleno desarrollo de la percepción de su cuerpo y su relación con el espacio y el tiempo. Estas habilidades son componentes esenciales del aprendizaje motor del niño y se reflejan tanto en las actividades escolares como en las cotidianas (17).

Por último, se debe reconocer que el desarrollo es un proceso continuo, a través del cual el niño adquiere habilidades cada vez más complejas, que le permiten inte $r$ ractuar con sus semejantes y responder de diversas formas a situaciones de su medio ambiente (18). Los niños de 4 a 8 años se encuentran en la fase de adquisición y fortalecimiento de movimientos fundamentales. En esta fase, el niño está activamente involucrado en la exploración y experimentación de la capacidad de movimiento de su cuerpo. Así que este tiempo es el apropiado para descubrir la forma de ejecutar una variedad de movimientos locomotores, manipulativos y de equilibrio. Los niños que no desarrollan patrones de movimientos maduros durante este período, pueden presentar, por ejemplo, dificultades en la realización de habilidades deportivas más complejas (19).

Durante la etapa escolar, los niños y niñas asimilan aspectos relacionados con su forma de entenderse y entender el mundo; entre estas se encuentran las formas de concebir los contactos corporales según diferentes culturas y géneros. De la misma manera, durante este tiempo, se refuerzan las capacidades físicas en ambos sexos, como la fuerza, la potencia y la resistencia, principalmente en el hombre; y la adquisición de destrezas como el ritmo, la coordinación, el equilibrio, o actividades como la danza y la expresión corporal, preferentemente en las mujeres (20). 


\section{Referencias}

1. Schonhaut BL, Rojas NP, Kaempffer R. Factores de riesgo asociados a déficit del desarrollo psicomotor en preescolares de nivel socioeconómico bajo, Comuna urbana rural Región Metropolitana, 2003. Rev, Chil Pediatr. 2005; 76 (6): 589-598.

2. González C, Cecchini JA, López J, Riaño C. Disponibilidad de las habilidades motrices en escolares de 4 a 14 años, Aplicabilidad del test de Desarrollo Motor Grueso de Ulrich. Aula Abierta. 2009; 37 (2): 19-28.

3. Baena A, Granero A, Ruiz PJ. Procedures and instruments for the measurement and evaluation of motor development in the educations system. J Sport Health Res. 2010; 2(2):63-76.

4. Zampa C. La importancia de respetar las etapas del desarrollo en deporte: una mirada desde la psicomotricidad. Rev. Electrón. Psicol. Polít. 2007; 5 (14): 0-0,.

5. Madrona PG, Contreras OR, Gómez I. Habilidades motrices en la infancia y su desarrollo desde una educación física animada. Revista Iberoamericana de educación. 2008 (47): 71-96.

6. Da Fonseca V. Manual de Observación Psicomotriz: Significación psiconeurológica de los factores psicomotores. Madrid: Publicaciones INDE; 1998.

7. Vidarte JA, Ezquerro M, Giráldez MA. Perfil psicomotor de niños de 5 a 12 años diagnosticados clínicamente de trastorno por déficit de atención/hiperactividad en Colombia. Rev. Neurol. 2009; 49 (2): 69-75.

8. Ruiz LM, Graupera JL, Competencia motriz y género entre escolares españoles. Rev. in. med. cienc. act. fís. deporte. 2003; 3(10): 101-111.

9. Bustamante A, Caballero L, Enciso N, Salazar I, Teixeira AF, et al. Coordinación motora: Influencia de la edad, sexo, estatus socioeconómico y niveles de adiposidad en niños peruanos. Rev. Bras. Cineantropom. Desempenho Hum. 2008; 10(1): 25-34.

10. Borda MI, Escudero CA, Escudero EL, Fuentes MJ, Gómez MJ, Jiménez MA, et al. Buenas prácticas en Educación y en Psicología. Una propuesta para educar en la igualdad. BOJA, 2006; 236: 17-49.
11. Sabagg A, Da Silveira RA, De Souza CA, Cardoso FL. Perfil de desenvolvimiento motor de escolares. Rev. Digital. 2010; 15(148): 0-0.

12. Campo LA, Jiménez PA, Maestre KM, Paredes NE. Características del desarrollo motor en niños de 3 a 7 años de la ciudad de Barranquilla. Psicogente, 2011; 14 (25): 76-89.

13. Cantó R, Ruiz LM. Comportamiento motor espontáneo en el patio de recreo escolar análisis de las diferencias por género en la ocupación del espacio durante el recreo escolar. Rev.int. cienc. deporte. 2005; 1(1): 28-45.

14. Garaigordobil M, Amigo R, Inteligencia: Diferencias de género y relaciones con factores psicomotrices, conductuales y emocionales en niños de 5 años. Interdisciplinaria, 2010; 27(2): 229-245.

15. Martínez EJ, Franco JC. Influencia de un programa de intervención psicomotriz sobre la creatividad motriz en niños de educación infantil. Bordón 2008; 60 (2): 107-121.

16. Madrona PG, Contreras OR, Gómez S, Gómez I. Justificación de la Educación Física en la educación infantil. Educ, educ. 2008; 11(2): 159177.

17. Medina J, Broio GK, Marques I. Desenvolvimento da organização temporal de crianças com dificuldades de aprendizagem. Maringa. 2006; 17 (1): 107-116.

18. Campo LA, Importancia del desarrollo motor en relación con los procesos evolutivos del lenguaje y la cognición en niños de 3 a 7 años de la ciudad de Barranquilla (Colombia). Salud Uninorte. 2010; 26 (1): 65-76.

19. Muñoz L. Evaluación de las habilidades motoras y crecimiento físico de los niños de un jardín infantil. Educación Física y De $p$ porte. 1991; 13 (1-2): 1-17.

20. Rodríguez H, García A. Asimilación de códigos de género en las actividades del recreo escolar. Revista Interuniversitaria de Formación del Profesorado. 2009; 64 (23,1): 59-72.. 